\title{
PDF und Schluss? Wissenschafts- und Buchgeschichte, 18.-20. Jahrhundert
}

Alrun Schmidtke

Julia Amslinger 2017: Eine neue Form der Akademie. ,Poetik und Hermeneutik' - die Anfänge. Leiden: Wilhelm Fink, brosch., 386 S., $€ 51,00$ ISBN 978-3-7705-5384-6

Alex Csiszar 2018: The Scientific Journal. Authorship and the Politics of Knowledge in the Nineteenth Century. Chicago, IL: The University of Chicago Press, geb. 368 S., \$45,00, ISBN: 978-0-22655323-8

Carlos Spoerhase 2018: Das Format der Literatur. Praktiken materieller Textualität zwischen 1740 und 1830. Göttingen: Wallstein, geb. 810 S., €49,90, ISBN: 978-3-8353-3103-7

What is the history of (scientific) books?

"What is the history of books?" fragte Robert Darnton (1982) und lieferte mit seinem so betitelten Artikel eines der Gründungsdokumente einer Kulturgeschichte des Buches. Auf seine monografische Publikationsgeschichte der Encyclopédie folgend (Darnton 1979), befasste sich Darnton hier mit den Veröffentlichungsvorgängen der Questions sur l'Encyclopédie von Voltaire und nahm dessen Unterstützung für zeitgenössische Raubkopierer seines Werkes zum Anlass, um über den Status von Autorschaft, über die Fluidität gedruckter Formate und damit zusammenhängende Möglichkeiten und Grenzen von Leseforschung zu reflektieren (Darnton 1982). 
Solche Reflexionen über Formate, ihr Verhältnis zu Inhalten sowie über Autorschaft in Printmedien erscheinen zum jetzigen Zeitpunkt besonders deshalb drängend, weil wir uns als Forschende permanent dazu aufgefordert sehen, digitale und printmediale Autorschafts- und Lektürepraktiken gegeneinander abzuwägen. Beinahe im Akkord hören bzw. meistens: lesen wir darüber, wie das Ende des Buchzeitalters heraufbeschworen wird (McLuhan 1968). ${ }^{1}$ So proklamierte jüngst die Kampagne einer großen Berliner Universitätsbibliothek "Ich will doch eigentlich nur das PDF!“ und richtete, ganz zu Recht, ihre Aufmerksamkeit auf die Ausmaße der Bezahlschranken für Zeitschriftenartikel. ${ }^{2}$ Digitalisierungs- und Open-Access-Euphorien richten sich aber längst nicht nur auf Zeitschriftenartikel, sondern auch auf ,das Buch' (Hagner 2014) - Grund genug, einen Überblick darüber zu erfragen, was eigentlich die Wissenschaftsgeschichte zur Rolle von Printmedien zu sagen hat und wie wir als HistorikerInnen zu gegenwärtigen Entwicklungen des Wissenschaftsverlagswesens Stellung beziehen können.

Fragen wir also: What is the history of scientific books?, so können wir auf eine stattliche Anzahl von Studien zurückgreifen, die sich - um nur einige Beispiele zu nennen - etwa mit Popularisierungsphänomenen, disziplinären Verlagslandschaften oder dem fachlichen Stellenwert bestimmter Formate wie dem Lehrbuch auseinandergesetzt haben. Forschungsimpulse sind wiederholt von frisch zugänglich gewordenen Verlagsarchiven ausgegangen, wenn diese von den ursprünglich bestandsbildenden Unternehmen an staatliche Archive oder Bibliotheken übergeben worden sind, oder WissenschaftlerInnen solche Bestände durch Projektförderung beforschen konnten. ${ }^{3}$

Lag ein Fokus zunächst auf dem Untersuchungsgegenstand wissenschaftlicher Autorschaft, haben Buchbiografien sowie Forschungen über Lektürepraktiken die Medialität, also die spezifische mediale Verfasstheit, gedruckter Wissensbestände in den Vordergrund gerückt und Autorschaft fest an Lektüreerfahrungen und -erwartungen der Gelehrten bzw. disziplinär verorteten Wissenschaftler geknüpft. ${ }^{4}$ Solche Kenntnis um Lese- und Bearbeitungsformen (etwa Exzerpieren, Annotieren, Sammeln, Aufreihen) führte mitunter zu großer verlegerischer Aufmerksamkeit für bestimmte Formate, wie Analysen über Entstehungs- und Benutzungszusammenhänge etwa von pflanzengeografischen Karten (Güttler 2014), über repräsentationstaugliche Geschichtsbücher (Nolte 2018) oder gedruckte Nachschlagewerke mit Rückbezug auf frühneuzeitliche Lektürepraktiken (Blair 2010) zeigen. 


\section{Formatforschungen}

Warum haben sich WissenschaftlerInnen in der Vergangenheit für oder gegen bestimmte Formate entschieden? Welchen Versprechungen sind sie - als Leser wie Autoren - gefolgt, wo erfuhren sie Ernüchterung, wo waren Misserfolge durch Arretierungen zu retten? Hierzu gehört etwa die Erwartung, ein erwünschtes Publikum (besser) zu erreichen. Inwiefern dabei die Publikationssprache teilweise hinderlich sein konnte, ist zuletzt in Paul Noltes (2018) Buchbiografie von Thomas Nipperdeys Deutscher Geschichte thematisiert worden und lässt sich mit einer parallelen Lektüre der großen Studien zu Geschichtsverlagen (Blaschke 2010) sowie zu Wissenschaftssprachen (Gordin 2015) noch besser verstehen. ${ }^{5}$ Wozu in Büchern publizieren, wozu in Zeitschriften, wozu in Sammelbänden - Antworten hierauf finden sich in den drei zu besprechenden Monografien.

Disziplinär ordnen sie sich einer STS-informierten Wissenschaftsgeschichte („social history of science“, Csiszar) bzw. einer historisch-epistemologisch orientierten Literaturwissenschaft (Amslinger; Spoerhase) zu. Sie eint ein gemeinsames Interesse an den Praktiken, die mit Büchern und konkurrierenden Printmedien zu bestimmten historischen Zeitpunkten verknüpft waren. Alle drei geben Antworten darauf, welche Anforderungen vom 18. Jahrhundert (Spoerhase) über das 19. (Csiszar) bis hin zum 20. Jahrhundert (Amslinger) mit spezifischen medialen Formaten verknüpft sein konnten. Sie bezeugen ein rezentes, nun auch wissenschaftshistorisch gerahmtes Interesse an der printmedialen Verfasstheit von Inhalten - einem Interesse an Formaten wie dem wissenschaftlichen Sammelband (Amslinger), dem literarischen Buch (Spoerhase) und der wissenschaftlichen Zeitschrift (Csiszar). In diesen drei Studien kommen jeweils auch konkurrierende und mitunter konvergierende Medien zum Tragen: Bei Amslinger sind dies Briefe sowie persönliche Zusammenkünfte in Gruppen, Csiszar beschreibt, wie Akademiesitzungen durch schriftliche Formate Vor- und Nachbereitung sowie konkurrierende Deutungen fanden, die wiederum auf die Gelehrtendiskussionen zurückwirkten. Spoerhase schließlich unternimmt eine radikale Dekonstruktion der „Ideologie des Buches“ und postuliert, ,das Buch' sei immer schon ein problematisches, prekäres Format gewesen (S. 45).

\section{Historische Buchforschung: Was ist ein Buch?}

Carlos Spoerhases Monografie Das Format der Literatur. Praktiken materieller Textualität zwischen 1740 und 1830 präsentiert eine grundständige 
Formatforschung und ist aus der Habilitation des Autors hervorgegangen. Anhand literarischer Texte befasst er sich mit Themen wie Verfertigung, Vervielfältigung, Zirkulation und Medienkonversionen. Epochentypisch lagen literarisches Schaffen und gelehrte Tätigkeit mitunter nah beieinander, sodass wissenschaftliche Formate explizite Erwähnung finden.

Der Autor beginnt mit einer fundamentalen Verfremdung, indem er ankündigt, das Buch sei selbst in seiner vermeintlich stabilsten Epoche im 18. Jahrhundert eine durchweg instabile Entität gewesen und habe seither als Chiffre für eine Vielzahl von Formaten gedient. Es sei „erst retrospektiv zu dem Medium gemacht [worden], das gegen diese anderen zerstreuenden Medien eine substantielle Vereinheitlichung einstmals geleistet haben soll“ (S. 26). Dieses Forschungsprojekt bezeichnet der Literaturtheoretiker als „philologisch motivierte[] historische[] ,Poetologie' der literarischen Buchform“ (S. 16), wobei die Feststellung, das Buch sei im Untersuchungszeitraum („der Goethezeit“) „ein Format gewesen, das viel problematischer und prekärer war, als es sich aus unserer heutigen Perspektive darstellt", eine immer wieder anklingende Erinnerung buch- und wissenschaftshistorischer Untersuchungen spiegelt (S. 45).

Diese - wie Spoerhase zugibt - „vielleicht etwas wortreich geratene[]“ Studie (S. 9) bietet einen geradezu monumentalen Fundus von Anknüpfungspunkten für wissenschaftshistorisch gerichtete Buchforschung. Sie umfassen das Buch als Grenzobjekt (boundary object), als periodisches Werk, als Sammlung, als Geschenk, nach objektbiografischem Ansatz und als Auslöser für Klassifikationsprobleme. Besonders die Abschnitte über Manuskriptdrucke und Manuskriptnetzwerke, auf denen, wie der Autor betont, später nachfolgende printmediale Praktiken aufbauten, sind im Kontext der oben skizzierten wissenschaftshistorischen Positionen zu Buchproduktions- und Lektürepraktiken aufschlussreich. Als Manuskripte zirkulierten etwa wissenschaftliche Texte wie Fichtes Grundlage der gesammten Wissenschaftslehre, ein Werk, das als „Handschrift für seine Zuhörer“ gedruckt und mit individueller Autorisierung des Verfassers an Interessenten abgegeben wurde (S. 116-117). In diesem Abschnitt schildert Spoerhase systematisch, in welcher Tradition Texte „als Manuskripte“ gedruckt worden sind - etwa, um Honorarverpflichtungen zu umgehen (S. 134), um „unautorisierte[r] Zirkulation“ entgegenzuwirken (S. 137), „handschriftliche[s] Kopieren[]“ zu umgehen (S. 137), um ein Werk vor der Kritik zu schützen (S. 136) oder um es nur einem ausgewählten Kreis zur „gemeinsamen Vervollkommnung" zur Verfügung zu stellen (S. 147).

Im Untersuchungszeitraum, so verdeutlicht die Analyse eindrücklich, überschnitten sich soziale Medienpraktiken, die wir mit mündlicher, handschriftlicher und gedruckter Kommunikation assoziieren. 


\section{Sammelbände und Interdisziplinarität, 1960er Jahre}

Eine neue Form der Akademie. ,Poetik und Hermeneutik' - die Anfänge von Julia Amslinger bietet eine absolut genüssliche Lektüre über einen programmatisch interdisziplinären Forschungsverbund in der frühen Bundesrepublik und dessen Mediengebrauch. Das aus einer literaturwissenschaftlichen Dissertation hervorgegangene Buch über die Forschungsgruppe „Poetik und Hermeneutik“ teilt sich in zwei Teile - einen analytischen Hauptteil, in dem die Autorin dem „Entstehungsprozess [geisteswissenschaftlicher] Werke“, nämlich der Sammelbände dieser Gruppe, nachgeht sowie einem Anhang mit Biografien, Brief- und Manuskriptauszügen und einer Liste von 543 „Briefe[n], die für die Arbeit an diesem Buch zur Kenntnis genommen wurden" (S. 373). Amslinger schildert die Geschichte eines Forschungsideals der Interdisziplinarität, das von der Forschungsgruppe aus Germanisten, Historikern und Philosophen mit Hilfe von Sammelbänden materialisiert wurde, und kontrastiert solche Idealvorstellungen geisteswissenschaftlichen Arbeitens mit den Forschungsrealitäten der Gruppenmitglieder, denen sie mit Hilfe der umfangreichen Korrespondenzen aus den Nachlässen Hans Blumenbergs und Hans Robert Jauß’ nachgeht. Die ideale „zwischenfachliche“ (so der Begriff für Interdisziplinarität der 1960er Jahre) Forschung war demnach Bestandteil einer programmatischen „Verwissenschaftlichung der Geisteswissenschaften“ (S. 18). Die Arbeit an den analytischen Begriffen und den theoretischen Konzepten bildete eine fächerübergreifende Basis für die gemeinsame Arbeit und stabilisierte die heterogen zusammengesetzte Personengruppe.

Mit der Gruppenarbeit lag der Fokus nicht auf den individuellen Leistungen und persönlichen Verfehlungen einzelner Mitglieder (darunter Exilanten ebenso wie Sturmbandführer in der Waffen-SS), sondern darauf, fortwährend im Austausch zu stehen, Begriffe zu hinterfragen und im „Dauergespräch“ (S. 135) an gemeinsamen Themen zu arbeiten. Das Verschwinden der Individuen setzte sich im materiellen Artefakt der $\mathrm{Pu}$ blikation fort: Die Sammelbände, finanziert aus Landes- und Drittmitteln, waren bibliografisch nur durch den Herausgeber markiert, einzelne Autoren hingegen verschwanden im Innenleben. Sie bestanden aus einem Vorlagenteil präzirkulierter Texte und den überarbeiteten, ergänzten Protokollen der Sitzungen. Vor den Sitzungen der Forschungsgruppe ließen die Teilnehmenden ihre Texte zirkulieren, sodass bei diesen Treffen lediglich kurze Einführungen durch die jeweiligen Autoren erfolgten. Das Format erscheint dabei in seiner Bedeutung für die wissenschaftliche Arbeit vor allem im Entstehungsprozess Relevanz zu besitzen, wohingegen das fertige Produkt als eine Zwischenstufe aufgefasst wurde, deren Buchförmigkeit vor allem symbolischen Wert, etwa gegenüber den Geldgebern, besaß. 
Dem Arbeitsideal zufolge sollten Geisteswissenschaften aber im „Dauergespräch" stehen, eine Diskussion demnach nie durch einen Sammelband besiegelt, sondern nur vorläufig festgestellt werden.

In der Konkurrenz um individuelle Aufmerksamkeit und kollegiale Anerkennung führte das gewählte Kommunikations(zwischen)produkt dann auch mindestens einen der Teilnehmer am Verbund zur Zerknirschung, denn die Art der Publikation bedeutete das Zurücktreten einzelner Autoren hinter die Gruppe. Blumenberg bedauerte die Konsequenzen dieses Unsichtbarmachens individueller Arbeitsanteile an den Texten, denn sie verhindere den sonst üblichen Schriftenaustausch in Form von Sonderdrucken. Anstatt in Fußnoten Erwähnung zu finden (und dann einen Sonderdruck erwarten zu dürfen), war die intellektuelle Mitarbeit an fremden Texten von Vornherein in den Verhandlungsteil, also die Sitzungsprotokolle, verwiesen, und dort, so Blumenberg, gleichsam „beerdigt“, weil sie „optisch in der Masse des Dargebotenen völlig verschw[a]nd[]“ (S. 156).

Erscheint die Verlags- und Buchhandelsperspektive zunächst ausgeklammert, weil Amslingers Analyse hauptsächlich auf die Wissenschaftlernachlässe rekurriert, lohnt es sich doch sehr, das Buch aus einer solchen Perspektive zu lesen. Ausführlich werden Schreibpraktiken, Lektüregewohnheiten, Erwartungen an mündliche und schriftliche Kommunikationsformate geschildert. In den wiedergegebenen Quellenzitaten ist etwa von der "Schäbigkeit des Verlegers" die Rede, der - hier in einem Brief von Blumenberg an Jauß - als „dreiste[r] Selbstfinanzierer“ von Druckkostenübernahmen profitiert habe (S. 219). Solche Zitate von und über den Verleger Wilhelm Fink finden sich am Schluss des Buches und im Quellenanhang. Sie sind geradezu als Aufforderung zu lesen, in künftigen Forschungen gezielt auch auf die buchhändlerische Marktsituation von Fächern wie der Germanistik in den 1960er Jahren einzugehen. Amslingers Analyse lässt jedenfalls vermuten, dass privatwirtschaftliche Interessen das Wohl und Wehe geisteswissenschaftlicher Forschung maßgeblich mitbestimmten, wenngleich hier noch offen bleibt, welchen Vorbildern diese Sammelbandpublikation nacheiferte und, kontrafaktisch, welche anderen Publikationskanäle den Akteuren zur Verfügung standen - denn ein „Dauergespräch“ ähnelt in frappierender Weise dem Periodizitätsideal anderer Wissenschaften, die sich zeitgleich gegen vermeintliche Endgültigkeit in Buchform wandten. ${ }^{6}$ 


\section{Französische und britische Zeitschriftenlandschaft im 19. Jahrhundert}

The Scientific Journal. Authorship and the Politics of Knowledge in the Nineteenth Century von Alex Csiszar befasst sich mit zahlreichen Konflikten der verschriftlichten wissenschaftlichen Kommunikation im 19. Jahrhundert, die anhand des Formats der wissenschaftlichen Zeitschrift und der darin publizierten Genres wie dem Zeitschriftenaufsatz dargestellt sind. Das Buch, hervorgegangen aus Csiszars Dissertation, fokussiert auf die Funktionserwartungen, die im Laufe des 19. Jahrhunderts in unterschiedlichem Maße an Zeitschriften und Zeitschriftenbeiträge herangetragen worden sind. „How did so much epistemic weight come to be loaded into this one format over all others?" fragt Csiszar gleich zu Beginn (S. 2) und konstatiert, "the public legitimacy of the scientific enterprise“, also die öffentliche Legitimität naturwissenschaftlicher Arbeit, sei im 19. Jahrhundert mit einem einzelnen Format wissenschaftlicher Literatur verknüpft worden, nämlich der Zeitschrift. Als Hauptakteure dieser Entwicklung macht er die wissenschaftlichen Akademien in Paris und London aus, deren Orientierung in Richtung periodischer Publikationen eine Entwicklung des 19. Jahrhunderts gewesen sei und die der Autor als Appropriationshandlung in Rückgriff auf bestehende publizistische Formate beschreibt, besonders kommerziell erfolgreicher Zeitschriften von Verlagen, die weder an eine wissenschaftliche Gesellschaft noch an eine Universität gebunden waren.

Csiszar entwickelt seine Studie hauptsächlich entlang britischer und französischer Zeitschriften - „Britain and France [are] a natural setting to focus on", denn, so Csiszar, das kontinentaleuropäische Zeitschriftenwesen habe „anders" funktioniert als das modellhaft aufgefasste westeuropäische (französische, britische) und US-amerikanische (S. 16). In Frankreich hätte sich mit Zeitschriftenneugründungen im 19. Jahrhundert vor allem die Hoffnung verbunden, das Monopol der Akademiemitglieder für die öffentliche Beurteilung von Erfindungen zu brechen, in Großbritannien habe der Schwerpunkt auf dem Demokratisierungspotenzial gelegen, das gegen das elitäre, geheimniskrämerische Vorgehen der Fellows of the Royal Society gerichtet war, und in anderen europäischen Ländern schließlich sei mit dem Format zuallererst ein Gemeinschaftspotenzial für räumlich getrennte Gruppen von Wissenschaftlern assoziiert gewesen (S. 44).

Die Pariser Académie des sciences übernahm die Begutachtung von Patenten und publizierte nur alle paar Jahre ein „Berichte“-Periodikum, sodass es Zeitungen und Zeitschriften oblag, wissenschaftliche Nachrichten abzudrucken. Csiszars Analyse zufolge wurden so politische Zeitschriften zu einem Kommunikationsmedium von Wissenschaftlern, die sich mit de- 
ren Hilfe etwa über Sitzungen der Akademien auf dem Laufenden hielten. Der Autor verknüpft diese Feststellung mit der expliziten Aufforderung, die Unterscheidung von ,Fachpublikum' und ,Laienpublikum', ,Fachzeitschrift' und ,Publikumszeitschrift' und dergleichen für das 19. Jahrhundert als nicht produktive analytische Kategorien fallenzulassen.

In Großbritannien war eine Mitgliedschaft in der Royal Society zunächst gar nicht an den Nachweis wissenschaftlicher Autorschaft gebunden, im Gegenteil, so Csiszar, sei Autorschaft lange Zeit mit der Notwendigkeit eines nichtaristokratischen Broterwerbs verknüpft gewesen und damit als anrüchig aufgefasst worden (S. 127). Orientierten sich französische wissenschaftliche Zeitschriften an den Genres der politischen Tageszeitungen, war in Großbritannien das Publikationsprogramm des Verlagsunternehmers Richard Taylor (später Taylor \& Francis) maßgeblich. Obwohl offenbar zahlreiche britische wissenschaftliche Gesellschaften auf Taylors Publikationsdienste zurückgegriffen haben (S. 79, 140), bleibt die Beschreibung dieses durch ihn offenbar dominierten "Marktplatzes“ vage (S. 119), für den zumindest eine Positionierung der britischen Universitätsverlage wünschenswert gewesen wäre. Hier setzt Csiszar möglicherweise die Lektüre von Adrian Johns' The Nature of the Book voraus (Johns 1998). Wirtschaftliche Einschätzungen sind im Kapitel „What Is a Scientific Paper?" am deutlichsten präsent, das zugleich auch mit vielen offenen Fragen aufwartet, etwa danach, wieso Beiträge aus Enzyklopädien zwar zu den auflagenstärksten Druckprodukten des britischen Buchmarkts gehörten (S. 199), die Royal Society jedoch in ihrem 1867 publizierten - und für Csiszars Argumentation maßgeblichen - Catalogue of Scientific Papers Enzyklopädieartikel kategorisch ausschloss (S. 228). Könnte es sein, dass nicht-britische Verlagshäuser sich in dieser Zeit besonders auf dem Markt der so ausgeschlossenen Handbücher, Enzyklopädien und Lexika hervortaten, wie Csiszar andeutet, aber nicht weiter problematisiert (S. 238)?

Besonders lesenswert ist das letzte Analysekapitel, „Access Fantasies at the Fin de Siècle“, in welchem der Autor bibliografische Arbeiten um 1900 auf gegenwärtige Open-Access-Debatten bezieht, deren beider Stoßrichtung er als „solutionism“ kennzeichnet: „[I]t has always been a delusion to suppose that any information technology can solve complex problems of knowledge“ (S. 287). Diese Hoffnung war und ist an Erwartungen geknüpft, verschiedene Funktionen in einem Format zu vereinen: „permanent archive“, „breaking news“, „public repository“, „exclusive dominion of experts“, „complete record“ und eine „painstakingly vetted selection“ (S. 2). In Csiszars Buch sind all diese Erwartungen angerissen und bieten eine Unmenge an Anknüpfungspunkten für weiterführende Forschungen zu Zeitschriften und anderen periodischen Publikationsformaten. 


\section{PDF und Schluss?}

Carlos Spoerhase macht nachvollziehbar, dass es ,das Buch` als unveränderliches Format womöglich nie gegeben hat, dass also auch nach Etablierung und Verbreitung des Buchdrucks handschriftliche Formate und damit assoziierte Textverfertigung, Sammlungs- und Lektüretechniken Anwendung fanden und den Umgang von Gelehrten und Verlegern mit Texten prägten. Alex Csiszar fokussiert in seiner Untersuchung auf wissenschaftliche Zeitschriften und kommt zu dem Schluss, dass Format und Genre im Laufe des 19. Jahrhunderts sich ständig an wirtschaftlich, politisch und gesellschaftlich veränderte Rahmenbedingungen anpassten und die wissenschaftlichen Gesellschaften (in London und Paris) bei medialen Veränderungen immer auf Wegbereiter außerhalb der Akademien zurückgriffen, dass diese Gesellschaften folglich solchen Markt- und Medienpluralismus zur Orientierung benötigten. Julia Amslinger demonstriert anhand der Forschungsgruppe „Poetik und Hermeneutik“, inwiefern deren sich geradezu revolutionär gebärdendes Forschungsprogramm der Interdisziplinarität mit dem Format des geisteswissenschaftlichen Sammelbands verbunden war. Eindrücklich zeigt die Untersuchung, wie die beteiligten Verbundforscher die Verfertigungsphase des Textprodukts nutzten, um miteinander in fachlichen Austausch zu treten: Die im Vorfeld organisierten Arbeitsgruppentreffen sowie die Vorstufen der Publikation machten dabei den Kern der wissenschaftlichen Arbeit aus und schienen mehr zur konzeptuellen Fachentwicklung beizutragen als die letztlich publizierten Bände.

Wie eingangs erwähnt, eint die vorliegenden Bücher ein gemeinsames Interesse an der medialen Verfasstheit von Inhalten. In Hinblick auf Robert Darntons Frage danach, was Buchgeschichte sei, könnte eine gemeinsame Antwort darauf schließlich lauten, dass buchhistorisch informierte Studien die Materialität fertiger Druckwerke nicht hinnehmen, sondern ihre Entstehungsgeschichten problematisieren und Kontexte ihrer Verfertigung konstruieren. Mit solch einer Historisierung regen sie dazu an, Formate pluralistisch aufzufassen, damit zugleich universellen Heilsversprechen mit kritischer Distanz zu begegnen. Die Art und Weise, wie Wissenschaftler Texte gelesen oder benutzt haben, etwa durch Zerschneiden (Blair), als Bearbeitungsvorlage (Güttler, Amslinger) oder im Tausch (Spoerhase, Csiszar), hatte Rückwirkungen darauf, welche Genres Verleger akzeptierten oder aktiv erbaten und welchen Formaten sie größere Chancen auf Verbreitung und Absatz einräumten. Im 20. Jahrhundert konnten solche Erwägungen zurückgestellt werden, wenn Finanzierungsmittel der Verbundforschung üppige Druckkosten trugen und weniger der publizierte Text die Diskussion anzuregen schien als vielmehr die im Vorfeld organisierten Arbeitsgruppentreffen und die Vorstufen der Publikation (Amslinger). 
Zugleich geben auch die Verlage der hier besprochenen Werke Antworten darauf, was aus ihren jeweiligen Perspektiven ein geisteswissenschaftliches Buch ausmacht. Während The University of Chicago Press (Csiszar) und der Wallstein Verlag (Spoerhase) materialreich geschützte Hardcoverbücher für die Rezension zur Verfügung stellten, versandte die Presseabteilung des Schoeningh Verlags - unter dem Dach der Brill-Gruppe anscheinend für Publikationen des Wilhelm Fink Verlags (Amslinger) zuständig - ein schreibgeschütztes PDF, in dem keinerlei digitale Annotationen möglich waren. Das mag praktisch sein, um die biografisch sortierten Bibliografien zu benutzen oder um in den umfangreich abgedruckten Quellen zu recherchieren. Einer Studie über wissenschaftliches Arbeiten und Publizieren die Buchförmigkeit abzuschlagen, verweist jedoch auf die Einstellung des Verlags zu dem von ihm publizierten Inhalt. Eine aufmerksame Lektüre, die eine solche geisteswissenschaftliche Arbeit verdient, so ergibt die Einzelanalyse im Gleichklang mit allen oben benannten Forschungsbeiträgen über das wissenschaftliche Arbeiten mit Büchern, ist an die gedruckte Form gebunden, bei der Blättern möglich ist, das gezielte Zur-Hand-Nehmen, Begreifen und nicht zuletzt die Benutzung eines Stiftes.

Eine Indizierung ist hier entfallen, während The University of Chicago Press im Buch von Csiszar zwar für ein solch hilfreiches Schlagwortverzeichnis gesorgt hat, dafür aber beschieden hat, eine solche Studie über Publikationen käme ohne Literaturverzeichnis aus. Schade ist auch, dass nicht schon aus dem Titel, sondern erst aus der Einleitung hervorgeht, dass die Analyse auf die französische Akademie der Wissenschaften und die Royal Society in London fokussiert. Dass die Wahl hier auf "The Scientific Journal" fiel, ist in Hinblick auf Csiszars durchgehenden Gegenwartsbezug zwar plausibel, den kritischen Apparat allerdings nicht mit einem Literaturverzeichnis auszustatten, ist ebenfalls bedauerlich, denn daraus ginge schnell hervor, dass nicht-englische und nicht-französische Publikationsperspektiven hier weitgehend außen vor geblieben sind.

Bei Satz und Ausstattung des Wallstein-Buches schließlich sollte, neben seinem massigen Umfang und seinem dementsprechenden, zur Schreibtischarbeit zwingenden Gewicht, vor allem die Qualität der Farbtafeln hervorgehoben werden. Diese farbigen Objektfotografien scheinen die Lesenden geradezu aufzufordern, schnellstmöglich eine Bibliothek mit alten Buchbeständen aufzusuchen anstatt auf gräuliche oder verpixelte Scans mit furchtbaren Text(v)erkennungen zurückzugreifen. „Ich möchte doch eigentlich nur das PDF" sollte, so muss das Fazit hier schließlich lauten, auf periodische Literatur, auf „Broken Pieces of Fact“ (wie Csiszars ursprünglicher Dissertationstitel 2010 lautete), beschränkt bleiben. Buchund wissenschaftshistorisch informiert sollten wir weiterhin zugestehen, 
dass sich Lektürepraktiken von periodischer Literatur von denen monografisch verfasster, geisteswissenschaftlicher Studien, wie den hier besprochenen, unterscheiden dürfen.

Hinweis des Verlags Der Verlag bleibt in Hinblick auf geografische Zuordnungen und Gebietsbezeichnungen in veröffentlichten Karten und Institutsadressen neutral.

\section{Anmerkungen}

1 Zu rezenten Abgesängen auf das gedruckte Buch, vgl. Hagner (2014).

2 Universitätsbibliothek der TU Berlin 2018. Open-Access-Materialien, URL: http:// www.ub.tu-berlin.de/publizieren/open-access/beratung-workshops-materialien/openaccess-materialien/ (04.01.2019).

3 Heumann (2014); Daum (2002); Estermann \& Schneider (2007); Remmert \& Schneider (2010); Daling (2011); Kaiser (2005); Badino \& Navarro (2013); Jaspers \& Paul (2018); Fyfe et al. (2017).

$4 \quad$ Biagioli \& Galison (2003); Holl (1996); Browne (2006); Secord (2000); Baldwin (2015); Felsch (2015); Mielewczik et al. (2017).

5 Vgl. hierzu auch die Rezension von Schneider (2018).

6 Ein historisches Plädoyer für Bücher findet sich wiederabgedruckt bei Amslinger (S. 128-129), nämlich Hans Robert Jauß’ äußerst unterhaltsame Studierendenbroschüre Besondere Hinweise, die Ihnen bei Beginn Ihres Studiums von Nutzen sein können.

\section{Literatur}

Badino, Massimiliano und Jaume Navarro (Hg.) 2013. Research and Pedagogy. A History of Quantum Physics Through Its Textbooks. Berlin: Edition Open Access.

Baldwin, Melinda 2015. Making 'Nature.' The History of a Scientific Journal. Chicago, IL: The University of Chicago Press.

Biagioli, Mario und Peter Galison (Hg.) 2003. Scientific Authorship. Credit and Intellectual Property in Science. London: Routledge.

Blair, Ann M. 2010. Too Much to Know. Managing Scholarly Information Before the Modern Age. New Haven, CT: Yale University Press.

Blaschke, Olaf 2010. Verleger machen Geschichte. Buchhandel und Historiker seit 1945 im deutsch-britischen Vergleich. Göttingen: Wallstein.

Browne, Janet 2006. Darwin's Origin of Species. A Biography. London: Atlantic Books.

Csiszar, Alex Attila 2010. Broken Pieces of Fact. The Scientific Periodical and the Politics of Search in Nineteenth-Century France and Britain. PhD Thesis, Harvard University.

Daling, Dorien 2011. Stofwisselingen. Nederlandse uitgevers en de heruitvinding van het natuurwetenschappelijk tijdschrift, 1945-1970 [„Stoffwechsel. Niederländische Verlage und die Neuerfindung der naturwissenschaftlichen Zeitschrift, 1945-1970“]. Groningen: Walburg Pers.

Darnton, Robert 1979. The Business of Enlightenment. A Publishing History of the Encyclopédie 1775-1800. Cambridge, MA: Belknap Press.

Darnton, Robert 1982. What Is the History of Books? Daedalus (111): 65-83.

Daum, Andreas 2002. Wissenschaftspopularisierung im 19. Jahrhundert. Bürgerliche Kultur, naturwissenschaftliche Bildung und die deutsche Öffentlichkeit 1848-1914. München: Oldenbourg. 
Estermann, Monika und Ute Schneider (Hg.) 2007. Wissenschaftsverlage zwischen Professionalisierung und Popularisierung. Wiesbaden: Harrassowitz.

Felsch, Philipp 2015. Der lange Sommer der Theorie. Geschichte einer Revolte 1960-1990. München: C.H. Beck.

Fyfe, Aileen, Kelly Coate, Stephen Curry, Stuart Lawson, Noah Moxham und Camilla Mørk Røstvik 2017. Untangling Academic Publishing. A History of the Relationship Between Commercial Interests, Academic Prestige and the Circulation of Knowledge. Bericht zum Arts and Humanities Research Council Project „Publishing the Philosophical Transactions: A Social, Cultural and Economic History of a Learned Journal, 1665-2015“. URL: https://zenodo.org/record/546100 (4.1.2019).

Gordin, Michael D. 2015. Scientific Babel. How Science Was Done Before and After Global English. Chicago, IL: The University of Chicago Press [Scientific Babel. The Language of Science from the Fall of Latin to the Rise of English. London: Profile Books 2017].

Güttler, Nils 2014. Das Kosmoskop. Karten und ihre Benutzer in der Pflanzengeographie des 19. Jahrhunderts. Göttingen: Wallstein.

Hagner, Michael 2014. Zur Sache des Buches. Göttingen: Wallstein.

Heumann, Ina 2014. Gegenstücke. Populäres Wissen im transatlantischen Vergleich (19481984), Wien: Böhlau.

Holl, Frank 1996. Produktion und Distribution wissenschaftlicher Literatur. Der Physiker Max Born und sein Verleger Ferdinand Springer 1913-1970. Frankfurt am Main: Buchhändler-Vereinigung.

Jaspers, Anke und Morten Paul 2018. Bibliografie [des Suhrkamp-Forschungskollegs]. URL: https://suhrkamp-forschungskolleg.de/wissenswertes/bibliographie/ (4.1.2019).

Johns, Adrian 1998. The Nature of the Book. Print and Knowledge in the Making. Chicago, IL: The University of Chicago Press.

Kaiser, David (Hg.) 2005. Pedagogy and the Practice of Science. Historical and Contemporary Perspectives. Cambridge, MA: MIT Press.

McLuhan, Marshall 1968. Die Gutenberg-Galaxis. Das Ende des Buchzeitalters, Düsseldorf: Econ [en.: The Gutenberg Galaxy. The Making of Typographic Man. Toronto: University of Toronto Press 1962].

Mielewczik, Michael, Darrel P. Francis, Bruno Studer, Michal V. Simunek und Uwe Hossfeld 2017. Die Rezeption von Gregor Mendels Hybridisierungsversuchen im 19. Jahrhundert. Eine bio-bibliographische Studie. Nova acta Leopoldina (413): 83-134.

Nolte, Paul 2018. Lebens Werk. Thomas Nipperdeys, Deutsche Geschichte'-Biographie eines Buches. München: C.H. Beck.

Remmert, Volker R. und Ute Schneider 2010. Eine Disziplin und ihre Verleger. Disziplinenkultur und Publikationswesen der Mathematik in Deutschland, 1871-1949. Bielefeld: Transcript.

Schneider, Michael C. 2018. Paul Nolte 2018: Lebens Werk. Thomas Nipperdey[s] Deutsche Geschichte [Rezension]. NTM (26): 477-478.

Secord, James A. 2000. Victorian Sensation. The Extraordinary Publication, Reception, and Secret Authorship of 'Vestiges of the Natural History of Creation'. Chicago, IL: The University of Chicago Press.

\author{
Alrun Schmidtke \\ Lehrstuhl für Wissenschaftsgeschichte (Gerda Henkel Stipendiatin) \\ Humboldt-Universität zu Berlin \\ Unter den Linden 6 \\ 10099 Berlin \\ Deutschland \\ alrun.schmidtke@hu-berlin.de
}

